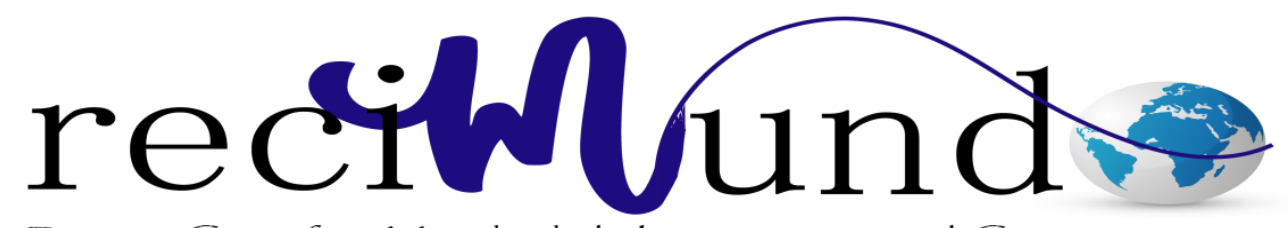

Revista Cientifica Mundo de la Investígación y el Conocimiento

Miriam Angélica Montalván Campoverde a ; Ana Margarita Ramos Maldonado b; Teresa Del Rocío Jaime Quimi ${ }^{\text {c }}$ : Lucila Duche Tacuri ${ }^{\mathrm{d}}$

El contexto sociocultural en la inteligencia narrativa de los menores

Revista Científica Mundo de la Investigación y el Conocimiento. Vol. 1 núm., 5, diciembre, 2017, pp. 625-649

DOI: 10.26820/recimundo/1.5.2017.625-649

Editorial Saberes del Conocimiento

a, b, c. Docentes de la Escuela de Educación Fiscal Región Amazónica. 


\section{EI contexto sociocultural en la inteligencia narrativa de los menores}

Vol. 1, núm. 5., (2017)

Miriam Angélica Montalván Campoverde; Ana Margarita Ramos Maldonado; Teresa Del Rocío Jaime Quimi; Lucila Duche Tacuri

\section{RESUMEN}

La presente investigación ha sido desarrollada por los docentes de la Escuela de Educación Fiscal Región Amazónica de la ciudad de Guayaquil. Mediante un análisis de la situación narrativa de los estudiantes. El objetivo es analizar el contexto que hace posible el desarrollo de la inteligencia narrativa para expresarse correctamente en forma oral o escrita. Se establece el contexto del aula, la influencia que sobre el Área de Lengua puede afectar directa o indirectamente el rendimiento de los estudiantes. El contexto de aprendizaje del niño es analizado desde la visión psicosocial en la que la familia y la escuela, son dos elementos de enorme influencia en la vida del estudiante de educación primaria. El niño de las Unidades Educativas fiscales del Ecuador se está educando en una realidad diferente a la de sus padres, como por ejemplo en la lucha por sus derechos, pero se descuida mucho la parte cognitiva, y en ese alcanzar el nivel deseado, se descuida la motivación, para que desde el hogar los contextos se manejen con sabiduría.

Palabras claves: Narrativa; sociocultural; infantes. 


\title{
El contexto sociocultural en la inteligencia narrativa de los menores
}

Vol. 1, núm. 5., (2017)

Miriam Angélica Montalván Campoverde; Ana Margarita Ramos Maldonado; Teresa Del Rocío

Jaime Quimi; Lucila Duche Tacuri

\begin{abstract}
The present investigation has been developed by the teachers of the Fiscal Education School of the Amazon Region of the city of Guayaquil. Through an analysis of the narrative situation of the students. The objective is to analyze the context that makes possible the development of narrative intelligence to express itself correctly orally or in writing. The context of the classroom is established, the influence that on the Language Area can directly or indirectly affect the students' performance. The context of the child's learning is analyzed from the psychosocial view in which the family and the school are two elements of enormous influence on the life of the elementary school student. The child of the Fiscal Education Units of Ecuador is being educated in a reality different from that of their parents, as for example in the struggle for their rights, but the cognitive part is neglected, and in that reaching the desired level, it is neglected the motivation, so that from the home the contexts are handled with wisdom.
\end{abstract}

Key words: Narrative; sociocultural; infants. 


\section{El contexto sociocultural en la inteligencia narrativa de los menores \\ Vol. 1, núm. 5., (2017) \\ Miriam Angélica Montalván Campoverde; Ana Margarita Ramos Maldonado; Teresa Del Rocío Jaime Quimi; Lucila Duche Tacuri}

\section{Introducción.}

Desde la existencia de instituciones educativas en las que existen directores de escuela que dan clases, y a su vez deben cumplir roles administrativos, como el hecho de tener escuelas en las que falta el wifi y el internet, como la realidad de la separación de los padres y el desempleo de uno de ellos, todos estos son condicionantes de una realidad que afecta el aprendizaje de los estudiantes.

Si hubiera una aula bien acondicionada como es que cada una de ellas tenga buenas instalaciones eléctricas porque ahora no las hay, y el hecho de que esas instalaciones durante más de 10 años son solo inventariadas, pero no le dan calidad en su servicio, limita las funciones así como la falta de conserjes en las escuelas es una necesidad que el estado debe satisfacer y no innovar con acuerdos que no se ajustan a la necesidad social del Buen Vivir. Además es necesario reconocer que desde el aula de clases faltan recursos y con ello, la comunicación está disminuida.

En relación al habla de los estudiantes, los niños varones son los que más utilizan un lenguaje vulgar, con malas palabras, para dirigirse a sus compañeros. Muchas veces lo hacen de manera normal, y llama mucha la atención a quienes los rodean. Se repite de manera aislada el caso de los representantes en el manejo del diálogo con los docentes, se expresan de manera exigente, deseando que la escuela les resuelva el mal comportamiento del estudiante, tarea que le corresponde al hogar, y muchas veces no dicen lo que sienten al maestro sino que lo hacen en las afueras de la institución. También hay una que otra madre que utiliza palabras inapropiadas y no tiene vergüenza de hacerlo ante los demás, lo que afecta la cultura de paz que el niño debe tener 


\section{El contexto sociocultural en la inteligencia narrativa de los menores}

Vol. 1, núm. 5., (2017)

Miriam Angélica Montalván Campoverde; Ana Margarita Ramos Maldonado; Teresa Del Rocío Jaime Quimi; Lucila Duche Tacuri

desde el hogar y que influye en la calidad de su rendimiento académico en dos áreas fundamentales, lengua y matemática que necesitan un contexto psicosocial para su aprendizaje.

A partir de la realidad económica de las familias, lo que se necesita es que sirva para conformar una cultura de estudio y de trabajo, pero a raíz de la realidad cultural de la familia, ésta influye muchísimo en el criterio y en la visión que de la vida tengan las personas. Muchos niños juegan de manera grosera en horas de recreo o dentro del aula, se golpean, se tiran al piso, se insultan; no existe preocupación en jugar de manera ordenada ni de respetar a los demás. A los varones en su mayoría les gusta jugar pelota, o jugar a la cogida pero en todo momento gritan o se insultan.

La situación de muchas familias en Guayaquil, ha evolucionado mucho desde 1970. Hoy la imagen de los abuelos constituye un pilar económico y logístico de enorme importancia en la realidad social. A la entrada de una escuela es común observar el siguiente cuadro familiar: el $40 \%$ de los estudiantes son llevados por las abuelas o abuelos, el $50 \%$ por las madres y el $10 \%$ por los papás. En ese ir y venir diario, se producen una serie de gastos que disminuyen los ingresos familiares, de ahí la importancia de la zonificación estudiantil de manera urgente y de la unificación de los hermanos para que estudien en una sola unidad educativa.

En la Escuela de Educación Básica Fiscal Región Amazónica de la ciudad de Guayaquil, se recibe a niños que en su mayoría pertenecen a la zona de las calles 15 , desde la $\mathrm{J}$ hasta la $\mathrm{M}$. Los oficios y profesiones de los representantes se dedican a diferentes labores como el de comerciantes, albañiles, carpinteros y en su mayoría eventuales. Las madres que trabajan son pocas las que dependen de una empresa donde ganan el básico. Siendo el porcentaje de niños 


\section{EI contexto sociocultural en la inteligencia narrativa de los menores}

Vol. 1, núm. 5., (2017)

Miriam Angélica Montalván Campoverde; Ana Margarita Ramos Maldonado; Teresa Del Rocío Jaime Quimi; Lucila Duche Tacuri

que viven sin sus dos padres del 30\%. La delincuencia y la drogadicción afecta a la convivencia de la zona. En esas condiciones los niños deben superar las condiciones más complejas en las que en sus hogares no tienen los espacios ni el clima organizacional adecuado que sea una prolongación

El rendimiento académico es un estado de condicionamientos continuos, en el que no existe una regla estática, pero sí elementos que pueden hacer de éste, todo un sistema nuclear. A partir de la realidad del aula, cada niño es un ser diferente para el aprendizaje. En el caso del Área de Lengua los elementos que intervienen en el aprendizaje no son los mismos que en el área de matemática, porque si en verdad ambos son significativos, el de matemática está cargada de mayor problematicidad.

Los estudiantes desde pequeños aprenden a identificar y usar de manera correcta los sustantivos, verbos y adjetivos, en cada grado se profundiza y hace práctica de éstos en oraciones y redacciones cortas. Pocos han logrado leer de manera clara y con entonación adecuada, respetando signos de puntuación y sin comerse letras ni palabras.

En la calidad de los escritos de los estudiantes se puede analizar y determinar si se han cumplido las competencias narrativas de los niños. Muchos padres y estudiantes no asocian la estética del escrito a la calidad del mismo. Sus afirmaciones constantes son: pero si está bien escrito, pero si está todo. Las formalidades no son tomadas en cuenta y ello puede llevar a hacer de las obras escritas solo elementos de interpretación subjetiva. A medida que los estudiantes tengan a bien hacer una obra, a ellos se les debe entregar una rúbrica que debe tener la venia y el 


\section{El contexto sociocultural en la inteligencia narrativa de los menores}

Vol. 1, núm. 5., (2017)

Miriam Angélica Montalván Campoverde; Ana Margarita Ramos Maldonado; Teresa Del Rocío Jaime Quimi; Lucila Duche Tacuri

respeto de las autoridades para implementarla y dar por asentado de qué forma el docente va a calificar.

\section{Desarrollo}

El matrimonio como la familia necesitan el aporte insustituible de un varón esposo y padre y de una mujer esposa y madre. La mujer y el varón tienen su propia personalidad según su naturaleza insustituible, y esas cualidades naturales enriquecen la vida en familia y la conciencia moral de los estudiantes.

Todos los niños mantienen círculos familiares, pero el tipo y clase de esos círculos son diferentes desde hace 20 años. La implementación del neoliberalismo en la educación ecuatoriana, no le permite llegar a la familia tradicional a acondicionar los valores tradicionales de la sociedad como son: la honestidad, la paz, el trabajo, la solidaridad, el respeto a los mayores, y otros.

De ese desarrollo, de la unión de los valores resurgen a veces, uniones maritales que logran formar una familia, en la que se respira el esfuerzo común por formar una sociedad material necesaria para la vida como es la construcción de una casa donde sea posible la convivencia y el ejercicio de los valores. La familia no se forma por esfuerzos personales sino conjuntos. Es una sociedad de bienes que necesita de valores afectivos como el cariño, la sonrisa, la paz y la bondad para hacer de la familia un sistema enriquecido con espíritu de grupo.

Con esa combinación de lo material y los valores, han resurgido durante años familias en el Ecuador, pero el condicionamiento permisivo de las leyes, no ayudan a que se materialice ese 


\section{EI contexto sociocultural en la inteligencia narrativa de los menores}

Vol. 1, núm. 5., (2017)

Miriam Angélica Montalván Campoverde; Ana Margarita Ramos Maldonado; Teresa Del Rocío Jaime Quimi; Lucila Duche Tacuri

sistema. La familia por lo tanto sufre mucho y naufraga en el intento de fomentar los valores, pues se puede encontrar con una realidad diferente en el aula, en la que los padres colaboran generalmente cuando se les insiste, pero cuando no, se ausentan de actividades que requieren la lucha continua para llevar procesos áulicos como las exposiciones o la exposición de proyectos.

La inteligencia emocional es una motivación externa que se necesita educar desde el hogar, por lo tanto deben volver dos elementos a las instituciones educativas, la escuela para padres y los centros de mediación que bien pueden existir por circuitos. De tal manera que desde el hogar los niños posean un equilibrio y una riqueza emocional que les permita obtener esquemas de aprendizaje de mejor calidad, de tal forma que se unan la inteligencia cognitiva con la inteligencia emocional. La violencia que muchos de los niños exponen y expresan en las aulas, se derivan el $80 \%$ desde el hogar y el $10 \%$ desde una respuesta a una ofensa y el otro $10 \%$ a las características psicosomáticas de los estudiantes.

Según (Clavijo, 2016)

La memoria no es todo lo que se ha vivido, sino lo que se puede recordar de ella para trasmitirla. Su carácter social la transforma en un producto de significados compartidos que reflejan acciones conjuntas de individuos en cada momento histórico. Los vehículos de la memoria tienen un rol muy importante para la comunicación de esta, movilizan representaciones, versiones del pasado, y una determinada identidad reinvidicada por un grupo social. ( Pág. 192) 


\section{El contexto sociocultural en la inteligencia narrativa de los menores}

Vol. 1, núm. 5., (2017)

Miriam Angélica Montalván Campoverde; Ana Margarita Ramos Maldonado; Teresa Del Rocío Jaime Quimi; Lucila Duche Tacuri

Existe un sentido de responsabilidad muy bajo entre la familia y sus niños para con la escuela, especialmente los niños desde el quinto, año de EGB a eso se despreocupan de presentar tareas y prepararse para las evaluaciones.

Existen muchos estudiantes que viven con sus abuelos y con familiares cercanos de padre o madre.

Dentro de las competencias que debe tener el niño en el área de lengua es el de la caligrafía que significa la belleza de la grafía. Las ventajas de una buena caligrafía es la capacidad que tiene lo material de la cadena hablada de poder ser inteligible a cualquier lector. En la medida que el rasgo superior, medio o inferior esté de acuerdo al código universal, en esa medida será posible su inteligibilidad. Una de las preocupaciones que debe acompañar a los padres es el lugar donde los estudiantes realizan las tareas. La mesa tiene que tener estabilidad en sus bases y debe estar limpia con la finalidad de que los cuadernos y libros no se manchen. Esa estabilidad estará dada por el nivel que debe tener el suelo con relación a la mesa o a la inversa.

El déficit de la atención en el área de lengua se debe a muchos factores. Si bien en el medio se puede tratar de una disfunción somática, en la realidad, lo que sucede es que muchos escritos no interesan a los niños por la mala calidad de los escritos y otras veces porque las lecturas seleccionadas no corresponden a su edad o experiencias previas.

Sin embargo la imaginación es lo que amerita en la edad infantil. Es notable su preocupación por la literatura fantástica. La capacidad de atención se manifiesta en ellos por lo que supone en sus vidas un ideal: Batman y Robin, Superman, en su momento han sido superados por: Spiderman, Capitán América, Thor, que representa la valentía, Hulk el personaje 


\section{EI contexto sociocultural en la inteligencia narrativa de los menores}

Vol. 1, núm. 5., (2017)

Miriam Angélica Montalván Campoverde; Ana Margarita Ramos Maldonado; Teresa Del Rocío Jaime Quimi; Lucila Duche Tacuri

de la ira, Ironman, el millonario, filántropo, y sarcástico, Dora la exploradora. Pero, ¿por qué estos personajes son los preferidos en los niños con déficit de la atención? Y ¿cómo, a pesar de estar fuera de su contexto social, se adaptan a su realidad individual? A partir de su realidad individual, cada personaje se revitaliza por el mundo interior que el niño está elaborando en su yo personal y le da la capacidad de interpretar cada una de esas actitudes.

El sistema educativo no aprovecha esa realidad del niño para insertar valores de la edad del estudiante, se vive una carestía de autores que adapten esos parámetros morales a la realidad ética que ya el niño va formando a partir de su razonamiento social que aunque todavía es mínimo, sin embargo vale la pena ir formando y fortaleciendo.

Los errores estéticos que se comenten en la narrativa deben ser minimizados desde el análisis funcional. A partir del dominio aplicado de cada una de las categorías gramaticales se puede llegar a establecer cómo y cuándo se deben utilizar las palabras en la sintaxis. El gran error de la forma de enseñar y el aprendizaje de la lengua es pensar que la linguiística aplicada se puede realizar solo a partir de la lectura, como pensar que quien sabe sumar es un dominador de las matemáticas.

Ricardo, es un estudiante muy inquieto, le gusta jugar fútbol, tiene algunos compañeros que lo siguen, hay que llamarle la atención muy seguida, le gusta andar con las vastas del pantalón dobladas, y los docentes lo regañan constantemente. La presentación de tareas es de lo más normal, pocos son aquellos que se esmeran en dibujar o pegar figuras para que su cuaderno quede bonito, otros por lo menos usan diferente color de esfero para mejorar la presentación. Siempre se les solicita que no descuiden la presentación de sus cuadernos. 


\section{El contexto sociocultural en la inteligencia narrativa de los menores}

Vol. 1, núm. 5., (2017)

Miriam Angélica Montalván Campoverde; Ana Margarita Ramos Maldonado; Teresa Del Rocío Jaime Quimi; Lucila Duche Tacuri

La unión de palabras en la cadena escrita o hablada merece una calidad $\mathrm{X}$ de conocimientos previos en el campo semántico, metafórico, de la narrativa y de una u otra manera sirven útilmente para la creación y expresividad negativa. Las corrientes que defienden las teorías de que la ortografía o la sintaxis no son necesarias no han podido con la estética del proceso que da como resultado una expresividad exquisita dentro del texto. Por todo esto es muy importante que los principios que gobiernan la gramática se digan estableciendo como necesarios. El año 2011, en Madrid se lanza la Nueva Gramática de la Lengua Española, y con ello el mundo recibe las nuevas formas de incorporar vocablos a la expresividad escrita y hablada. Ante este nuevo escenario, pocos son los que saben cómo llegar a los niños, la nueva forma de abordar la narrativa.

Sarita, es una niña que se expresa de manera clara, siguiendo un orden lógico en sus ideas, es muy ordenada en sus tareas y le gusta mucho leer. Vive con su mamá y sus hermanos, trabaja en un mercado pero siempre está pendiente de lo que necesite Sarita en el grado.

No todos los estudiantes logran redactar de manera clara y ordenada, se comen palabras, no coordinan las ideas, y lo que es peor se pierden de lo que al inicio se les solicita que hagan. Ya en la expresividad verbal, la vida cotidiana es fundamental, y de allí el hogar junto con el barrio, representan el $60 \%$ del lenguaje verbal y no verbal del niño. Es lo que lleva a la escuela. Pero los contenidos de las asignaturas son condicionantes del lenguaje para ellos. Se establecen entonces procesos de lingüística cultural en el aula. Todas las zonas del país se pueden representar lingüísticamente en el aula, pero no. A partir de la nueva realidad el estudiante va asumiendo nuevos términos, vocablos, grupo de palabras de las cuales lo peor es cuando esas palabras adquieren significatividad para su corta edad. 


\section{EI contexto sociocultural en la inteligencia narrativa de los menores}

Vol. 1, núm. 5., (2017)

Miriam Angélica Montalván Campoverde; Ana Margarita Ramos Maldonado; Teresa Del Rocío Jaime Quimi; Lucila Duche Tacuri

La lingüística cognitiva puede dar luces de este fenómeno. El lenguaje no está fuera de la esfera social de los niños. Los niños categorizan sus realidades y cobran sentido las palabras. El currículo crea prototipos científicos que son los que muchas veces evitan que el desarrollado de las malas palabras se materialice.

El lenguaje humano es muy sensible a la categorización cognitiva y el lenguaje abstracto de los niños puede estar categorizado sin embargo no logra conceptualizar ciertos procesos. La utilización de metáforas conceptuales ya están en el niño como en palabras como: chéveres, ese man, ese pana, oye, pelada. Esas metáforas conceptuales son las que adquieren significados en los contextos de uso. Cuando en las familias estos estereotipos se dan casi siempre, se hace cultura con ellos, esas metáforas adquieren un valor social anexo a la familia que luego se socializa en la escuela.

La falta de ayuda del docente dentro del aula, ocasiona en los estudiantes un quemeimportismo al momento de dar lecciones o presentar un trabajo de manera oral o escrito. Si no se exige en todas las áreas por igual, no se puede conseguir los frutos al final de un quimestre o período lectivo. Los estudiantes necesidad de estrategias para adentrarse al mundo de la creación, caso contrario no podrá expresar nada en ningún momento.

A pesar de que no existe una relación demostrable de que la formación de los padres incida en la cultura lectora de los niños, sí existen indicios que la cultura familiar predispone a los estudiantes a apetecer o no la lectura, sin embargo leer sigue siendo un placer individual propio de la aprehensión de cada uno de ellos, y en cuestión de placer estético cada persona impera sobre sus propios gustos. 


\section{El contexto sociocultural en la inteligencia narrativa de los menores}

Vol. 1, núm. 5., (2017)

Miriam Angélica Montalván Campoverde; Ana Margarita Ramos Maldonado; Teresa Del Rocío Jaime Quimi; Lucila Duche Tacuri

El ano 2017 Nina Crespo, Walter Koza y Eva Sotelo hicieron una investigación acerca de la Complejidad sintáctica y construcción de narración. Análisis de una tarea de recontado en ninos de preescolar desde la Universidad Católica de Valparaíso en Chile. Entre sus observaciones, ellos deducen que: (Crespo, 2017)

Los estudios que abordan el fenómeno de la complejidad sintáctica tienen ya varios anos. Por un lado, se pueden citar los basados en la propuesta generativa que remiten estudios donde el foco está puesto en ver cómo los ninos actualizan su gramática universal y el diseno metodológico de estas investigaciones que se basan en el testeo de la capacidad del sujeto para discriminar significados a partir de pistas sintácticas, con lo cual, a partir del análisis de las características lingüísticas y cognitivas de las contingencias que afrontan los ninos en el proceso de comprenderlas y producirlas, según los parámetros de la gramática adulta, se podría conocer la actualización de los parámetros que, en la GU, condiciona las relaciones de complejización sintáctica. ( Pág. 23)

El gusto estético por la lectura es el plus que convierte al niño de escasos recursos económicos a ser tan lector como uno que tiene en su casa todos los elementos materiales como libros, pupitre personal y computadora que facilitaría el aprendizaje lector. Es entonces el gusto estético lo único que rompe las leyes de la economía y de todas las teorías psicológicas y es capaz de convertir a cualquier estudiante en una gran lector a partir de su inclinación personal y particular de leer, al ser una experiencia personal enriquecedora que lo lleva a leer cuantas veces desee sin necesidad de control, recomendación o consejo. 


\section{EI contexto sociocultural en la inteligencia narrativa de los menores}

Vol. 1, núm. 5., (2017)

Miriam Angélica Montalván Campoverde; Ana Margarita Ramos Maldonado; Teresa Del Rocío Jaime Quimi; Lucila Duche Tacuri

El hecho de que los padres no tengan títulos de tercer o cuarto, no significa que los niños sean estudiantes excelentes. Muchos padres ayudan a sus hijos al nivel que ellos pueden, y las responsables están pendientes hasta de ir a averiguar qué deben presentar sus hijos. Sin embargo el conocimiento es un hecho único, nadie puede aprender por otro, es un hecho que si bien amerita ayuda del otro, ya en su estructura cognitiva, cada quien arma su propio andamiaje mental. Por lo tanto el goce y disfrute del conocimiento puede ser grupal, pero cada uno es responsable de su propio disfrute y satisfacción. Según (Crespo, 2017) “El desarrollo del lenguaje durante la edad escolar, a partir de los 5 años, se inicia con un proceso de complejización sintáctica que puede ser descrita desde diferentes puntos de vista”. (Pág. 3)

Se da el caso en que algunos estudiantes que teniendo a los padres con un buen recorrido en la vida académica de tercero o cuarto nivel no están predispuestos para la lectura, y una de las causas aparentes es que cuando se les hace las tareas a los hijos para quedar bien ante sus compañeros y docentes, la tarea está bien, pero los niños no están bien, y avanzan en su vida académica sin las base teóricas que se necesita para avanzar en lo curricular. Lo mismo sucede en la vida profesional de hijos que han heredado los puestos administrativos y docentes de sus padres, no siempre cumplen con las expectativas como profesionales.

También se han dado casos, aunque muy pocos, en los que muchos profesionales tienen palabras y costumbres indecentes en su forma expresiva. Sin embargo, los padres constituyen la cultura que el niño trae a la escuela y que el currículo se encargara de mejorarla, aunque no siempre sea así. Lo que se puede concluir y deducir de este análisis experimental es que el placer estético le pertenece a cada lector y es un lazo dialéctico y motivador entre el autor y quien lo lee, y es individual y subjetivo. 


\section{El contexto sociocultural en la inteligencia narrativa de los menores}

Vol. 1, núm. 5., (2017)

Miriam Angélica Montalván Campoverde; Ana Margarita Ramos Maldonado; Teresa Del Rocío Jaime Quimi; Lucila Duche Tacuri

La calidad narrativa merece de los escritores, dos condiciones: el arte de describir en el tiempo situaciones y realidades y la combinación perfecta de lo que se escribe, según la sintaxis. Existen otros elementos necesarios como es el dominio del léxico y la descripción de las categorías con la combinación pictórica que merece el relato, sea breve o no.

Los niños que vienen de hogares donde se les inculca la responsabilidad tienen facilidad de expresar sus emociones y puntos de vista, así como de redactar sobre un tema de interés con amplitud. Pero se da también el caso de madres con enorme carga emotiva y amorosa sobre sus hijos, que no tienen estudios de tercer nivel y que logran que sus hijos aprovechen al máximo el gusto por el estudio. Lo mismo sucede con los padres que acompañan a los estudiantes varones a ese mundo de las letras o de las matemáticas y los reconfortan con su compañía y logran hacer de sus hijos grandes matemáticos o lectores. El mundo de los lectores, músicos o matemáticos sigue dependiendo del gusto estético.

\section{Metodología}

Se ha realizado un muestreo estratificado con 10 docentes de Educación Básica. 


\section{El contexto sociocultural en la inteligencia narrativa de los menores}

Vol. 1, núm. 5., (2017)

Miriam Angélica Montalván Campoverde; Ana Margarita Ramos Maldonado; Teresa Del Rocío Jaime Quimi; Lucila Duche Tacuri

\section{Análisis de los resultados}

\section{1.- Los niños con padre tienen mejor rendimiento académico}

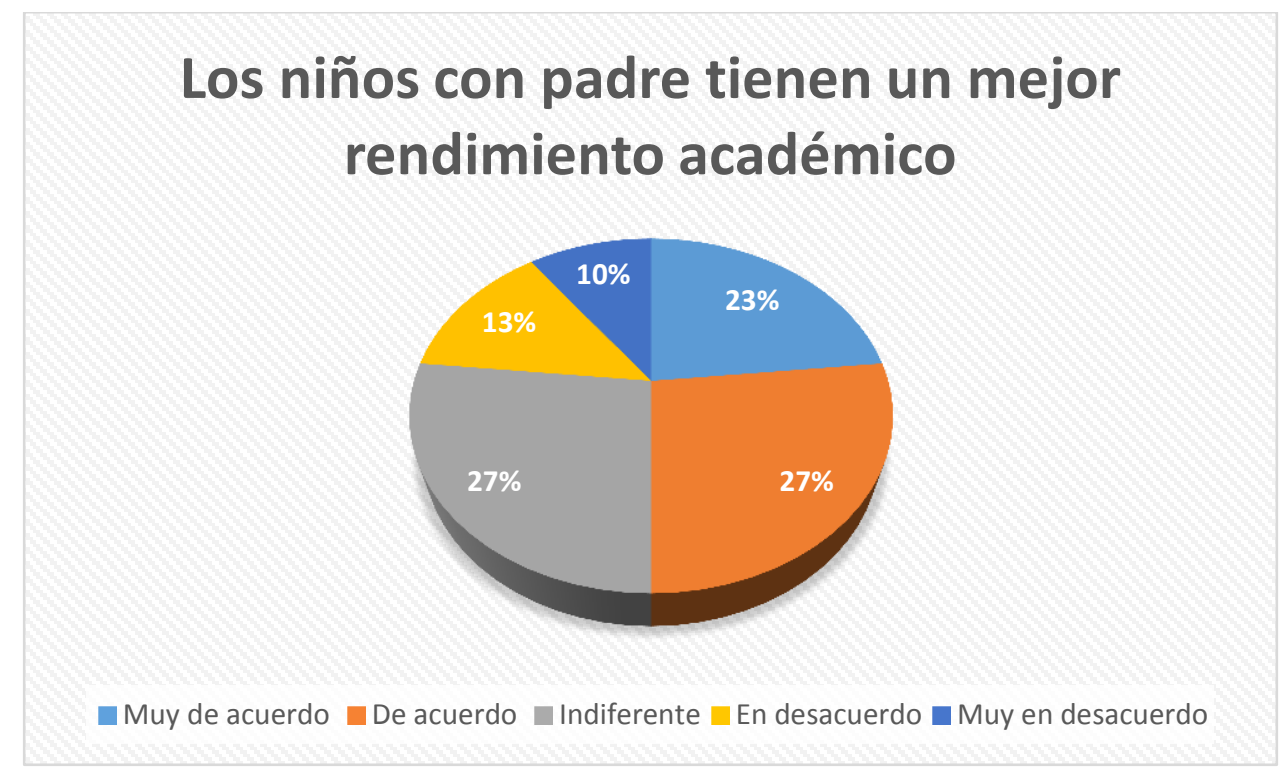

La imagen de la familia estructurada por padre y madre sigue siendo vital en la lectura de la realidad del aula, para establecer el rendimiento académico, de tal manera que a partir del desarrollo de esa inteligencia emocional, se puede lograr mejor concentración y entrega a los procesos áulicos y fuera de ellos. 
El contexto sociocultural en la inteligencia narrativa de los menores

Vol. 1, núm. 5., (2017)

Miriam Angélica Montalván Campoverde; Ana Margarita Ramos Maldonado; Teresa Del Rocío Jaime Quimi; Lucila Duche Tacuri

\section{2.- La realidad de la familia incide en la calidad de los escritos de los estudiantes.}

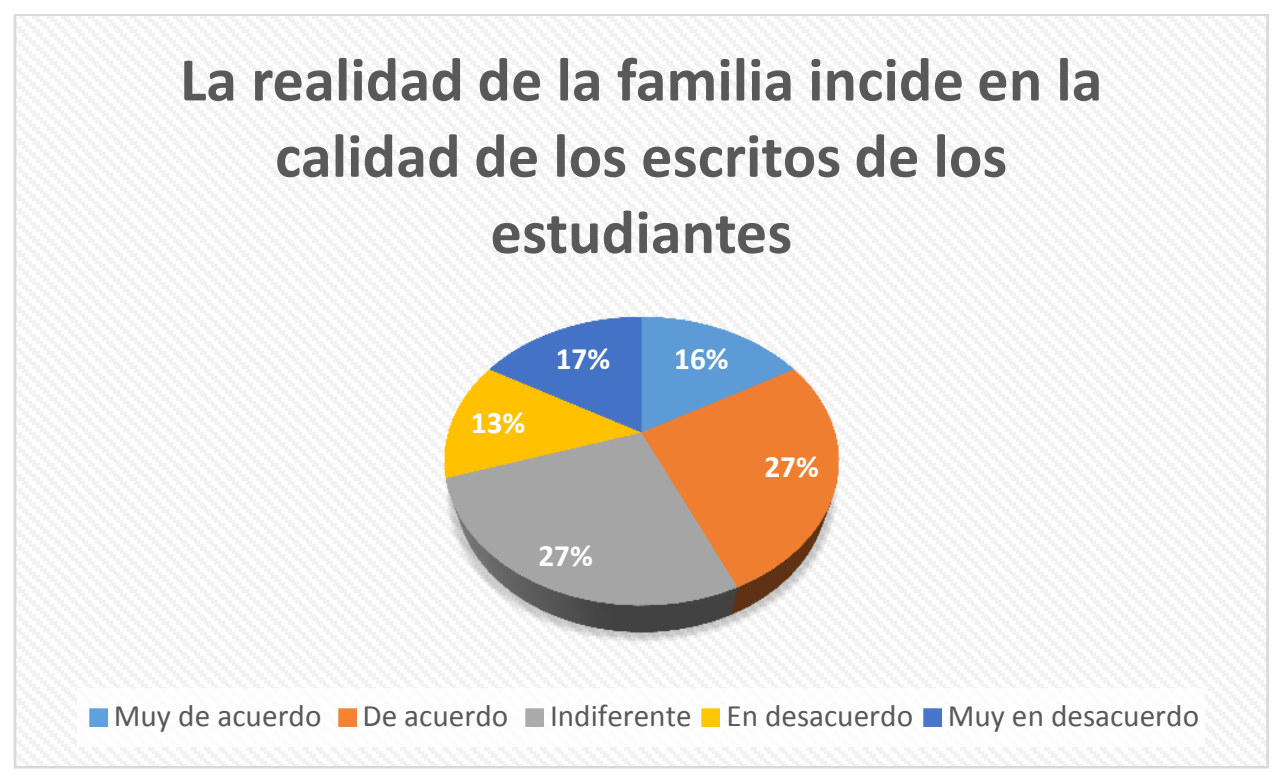

Una vez que se desarrolla la lectoescritura los niveles de redacción son diferentes y su dosificación y calidad hacen de la narrativa una cualidad literaria que debe ser competente desde la escuela, para ello es vital un nivel de contexto en los hogares, desde la mesa en la que trabajan, hasta la amplitud para la escritura que tengan en el aula. 


\section{El contexto sociocultural en la inteligencia narrativa de los menores}

Vol. 1, núm. 5., (2017)

Miriam Angélica Montalván Campoverde; Ana Margarita Ramos Maldonado; Teresa Del Rocío Jaime Quimi; Lucila Duche Tacuri

3.- La formación académica de los padres incide en la calidad del vocabulario de los estudiantes

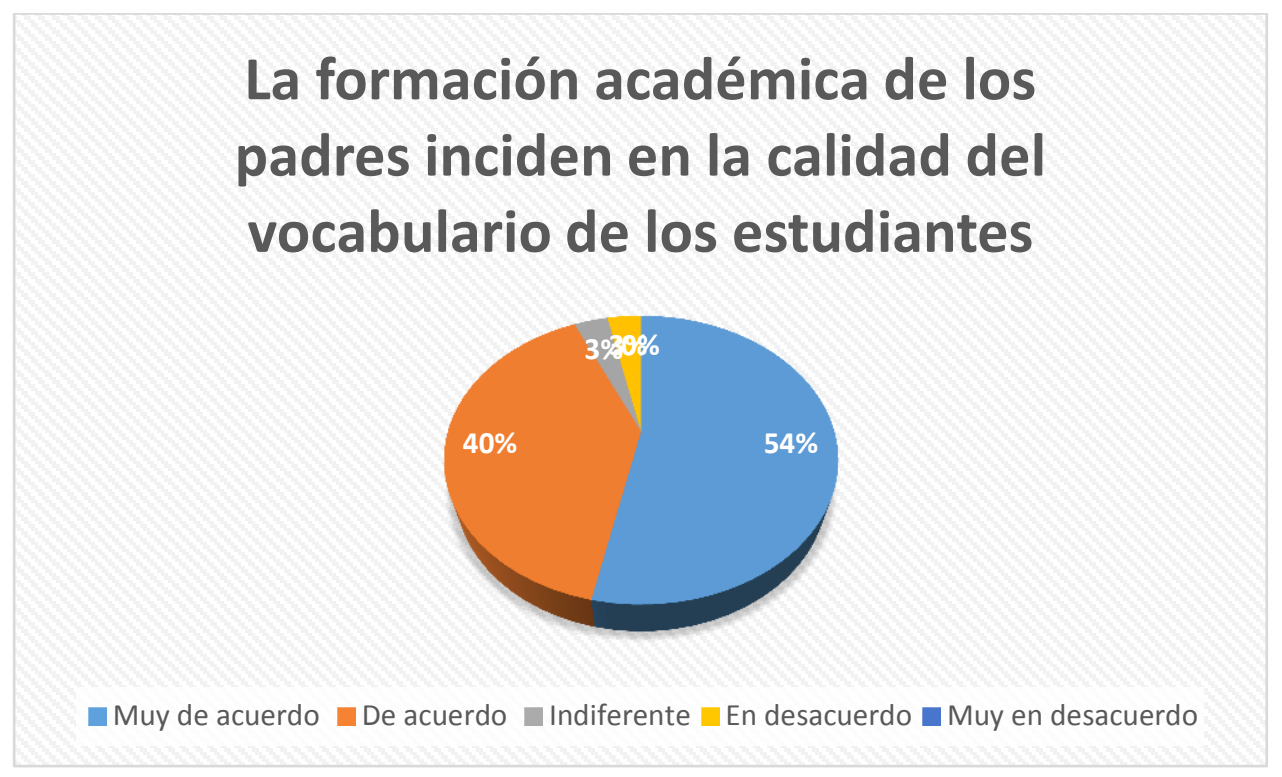

Sí incide en la calidad del vocabulario, ellos llevan palabras que se contextualizan y manejan en sus casas, en sus hogares y la clase se constituye en un apéndice que hace posible la nueva realidad del aula, pero para ellos esa realidad la convierten en la misma realidad, la que han elaborado sus padres. 
El contexto sociocultural en la inteligencia narrativa de los menores

Vol. 1, núm. 5., (2017)

Miriam Angélica Montalván Campoverde; Ana Margarita Ramos Maldonado; Teresa Del Rocío Jaime Quimi; Lucila Duche Tacuri

\section{4.- Los niños con déficit de la atención mantienen errores en la caligrafía.}

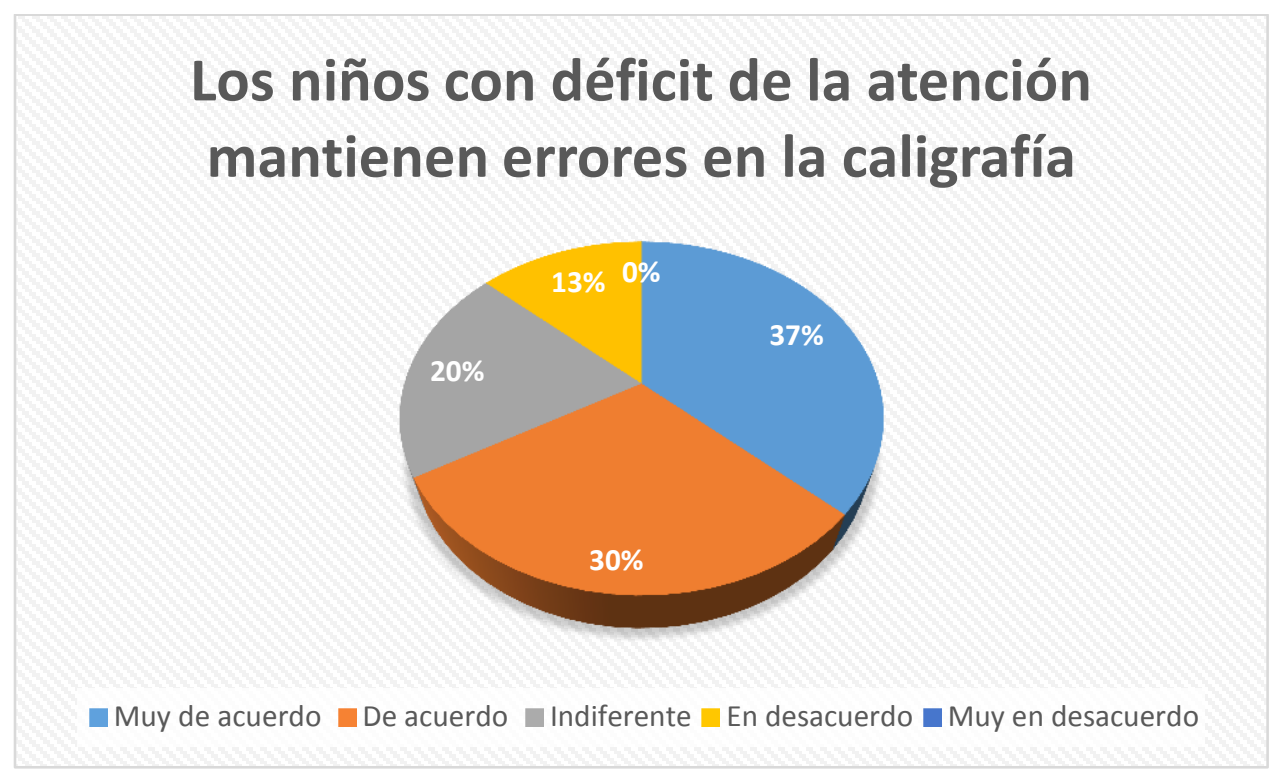

En su mayoría los niños con déficit de la atención entregan tareas de caligrafía con alto nivel de ilegibilidad, mantienen niveles en los cuales se salen de los parámetros establecidos como es el caso de las líneas, problemas con la tildación, y por lo tanto se complica el reconocer lo que ellos quieren expresar. 


\section{El contexto sociocultural en la inteligencia narrativa de los menores}

Vol. 1, núm. 5., (2017)

Miriam Angélica Montalván Campoverde; Ana Margarita Ramos Maldonado; Teresa Del Rocío Jaime Quimi; Lucila Duche Tacuri

5.- La capacidad narrativa permite la unión correcta de las palabras.

La capacidad narrativa permite la unión correcta de las palabras

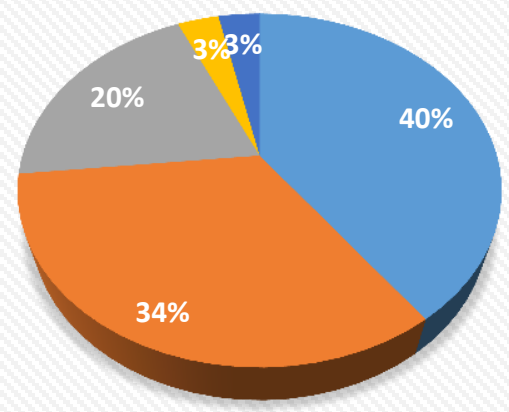

Muy de acuerdo $\square$ De acuerdo $\square$ Indiferente $\square$ En desacuerdo $\square$ Muy en desacuerdo 


\section{El contexto sociocultural en la inteligencia narrativa de los menores}

Vol. 1, núm. 5., (2017)

Miriam Angélica Montalván Campoverde; Ana Margarita Ramos Maldonado; Teresa Del Rocío Jaime Quimi; Lucila Duche Tacuri

6.- Los niños con déficit de la atención en su presentación escrita tienen deficiencias estéticas.

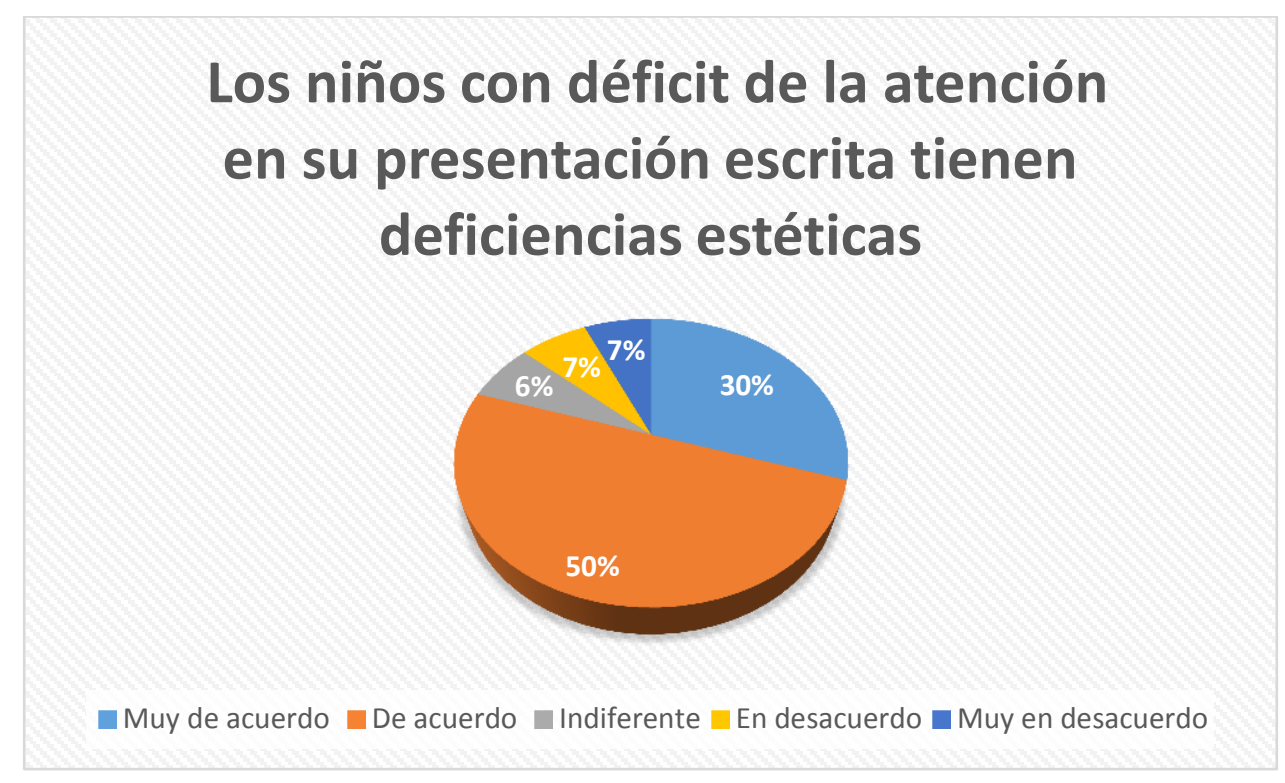

Sí, los niños con déficit de la atención tienen problemas en la calidad de los escritos, a diferencia de los que realizan los deberes con la guía de los padres que son profesionales o que están en la universidad, ellos son un soporte para el trabajo de los estudiantes, se convierte en una desventaja cuando les hacen las tareas o no les hacen razonar sobre los nudos en la zona de desarrollo próximo. 


\section{El contexto sociocultural en la inteligencia narrativa de los menores}

Vol. 1, núm. 5., (2017)

Miriam Angélica Montalván Campoverde; Ana Margarita Ramos Maldonado; Teresa Del Rocío Jaime Quimi; Lucila Duche Tacuri

7.- La genética del hogar incide en la calidad verbal de los estudiantes y sus palabras.

\section{La genética del hogar incide en la calidad verbal de los estudiantes y} sus palabras

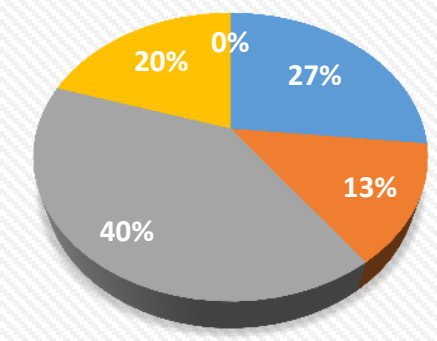

Muy de acuerdo $\square$ De acuerdo $\square$ Indiferente $\square$ En desacuerdo $\square$ Muy en desacuerdo

La genética del hogar se le llama en este proyecto a la combinación cultural del padre y de la madre, quienes logran amalgamar sus ideas para estructurar la cultura familiar que incide en la calidad verbal de los estudiantes. 


\section{El contexto sociocultural en la inteligencia narrativa de los menores}

Vol. 1, núm. 5., (2017)

Miriam Angélica Montalván Campoverde; Ana Margarita Ramos Maldonado; Teresa Del Rocío Jaime Quimi; Lucila Duche Tacuri

\section{8.- La calidad de la ortografía se asocia a varios factores como:}

\section{La calidad de la ortografía se asocia a varios factores como:

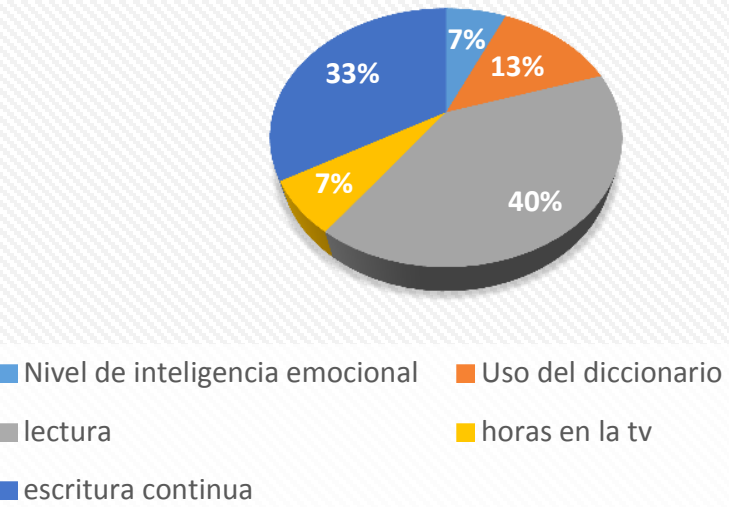

La lectura es el elemento que más influye en la calidad de la ortografía de los estudiantes, junto con la escritura continua, seguida del uso del diccionario y del nivel de inteligencia emocional. 


\section{EI contexto sociocultural en la inteligencia narrativa de los menores}

Vol. 1, núm. 5., (2017)

Miriam Angélica Montalván Campoverde; Ana Margarita Ramos Maldonado; Teresa Del Rocío Jaime Quimi; Lucila Duche Tacuri

\section{Docentes de la escuela de educación fiscal región amazónica}

\section{Conclusiones}

Los padres de familia que colaboran con la escuela, son los que tienen mayor preocupación por sus hijos.

La irresponsabilidad de los padres muchas veces es completa: en el control de tareas de los estudiantes, en la colaboración con la escuela y en la vida personal.

La narrativa mejora con el nivel académico de los padres, no así la lectura que es más orientada según el nivel de afectividad a las letras sea de cualquier nivel al que pertenezcan los padres.

El análisis de la lectoescritura se hace mejor desde la óptica semántica del diccionario.

Los hermanos mayores y los padres son de gran apoyo para los niños que comienzan en el proceso de escritura y lectura, pero también en la forma cómo aparecen a relatar.

\section{Bibliografía.}

Álamo, F. (11 de 11 de 2014). El microrrelato. Análisis, conformaciónn y función de sus categorías narrativas. Recuperado el 3 de 2 de 2017, de http://webs.ucm.es/info/especulo/numero42/microrre.html

Clavijo, P. (2016). ¿Qué es la verdad? ¿Qué es la ficción? Memorias movilizadas en la película . Revista Colombiana .

Crespo, O. \&. (2017). Complejidad sintáctica . Elae Círculo .

Cruz, M. (2016 ). Resignación de saberes ancestrales en la escuela . Plumilla Educativa . 


\section{El contexto sociocultural en la inteligencia narrativa de los menores}

Vol. 1, núm. 5., (2017)

Miriam Angélica Montalván Campoverde; Ana Margarita Ramos Maldonado; Teresa Del Rocío Jaime Quimi; Lucila Duche Tacuri

Martínez, J. (2016 ). Pensamiento permitido, formas de conocimiento y jerarquización del saber: apuntes psicosociales. Barcelona: Universidad de Barcelona .

Montero, V. (2016). Presupuesto metodológico en la enseñanza de adultos. Educar.

Obando, L. (2017). Semiótica Cognitiva y multimodalidad en la interacción pedagógica. ASSI .

Palacios, J. (2017 ). Descripción de las características de personalidad y dimensiones socioculturales en jóvenes mexicanos . Revista de Psicología. Vol. 35 .

Zabala, J. (2017). Relaciones textuales paar textuales de un afamado Sainete. Revista Científica de Cine y Fotografía, 133- 137. 